\title{
Telemedicine versus Physical Examination in Patients' Assessment during COVID-19 Pandemic: The Dubai Experience
}

\author{
Amr Elhennawy $^{\mathrm{a}}$ Fateh Almohammed Alsalem $^{\mathrm{b}} \quad$ Salah Bahric $^{\mathrm{c}} \quad$ Noor Alarfaj $^{\mathrm{d}}$ \\ aOperation Stream, COVID-19 Command \& Control Center, Dubai Nuclear Medicine and Molecular Imaging Center, \\ Clinical Support Services, Dubai Hospital, Dubai Health Authority, Dubai, UAE; ${ }^{b}$ ENT Section, Dubai Hospital, Dubai \\ Health Authority, Dubai, UAE; ' Operation Stream, COVID-19 Command \& Control Center, General Services and \\ Administration Affairs Department, Dubai Health Authority, Dubai, UAE; ${ }^{d}$ Operation Stream, COVID-19 Command \& \\ Control Center, Case Management Team, Dubai Health Authority, Dubai, UAE,
}

\section{Keywords}

Emergency · Infectious · Medicine · Preventive ·

Telemedicine · Dubai experience · Dubai Health Authority · COVID-19 pandemic $\cdot$ Triage

\begin{abstract}
Introduction: With the large number of cases during the $\mathrm{CO}$ VID-19 pandemic, a smart tool was urgently needed to handle all the positive cases at proper time such as using the technology and implementing telemedicine for triage. $\mathbf{O} \boldsymbol{b}$ jective: The purpose of this study is to detect the accuracy and effectiveness of telemedicine as an assessment tool for triage compared to physical assessment. Methods: In a random sample of 1,086 COVID-19 positive cases during the pandemic in Dubai, all the patients were initially assessed by trained staff using telemedicine following a standardized guidelines and accordingly action was taken by sending the patient to an isolation facility or for hospital admission; the second phase of assessment was conducted physically at the facility. We compared the accuracy of assessment by telemedicine encounter versus physical assessment by detect-
\end{abstract}

karger@karger.com www.karger.com/dmj

Karger $\stackrel{\text { ' }}{=}$
(C) 2021 The Author(s)

Published by S. Karger AG, Basel

This article is licensed under the Creative Commons AttributionNonCommercial-NoDerivatives 4.0 International License (CC BYNC-ND) (http://www.karger.com/Services/OpenAccessLicense) Usage and distribution for commercial purposes as well as any distribution of modified material requires written permission. ing any changes of the initial action within $12 \mathrm{~h}$. Results: Telemedicine was accurate in 1,080 out of 1,086 screened patients (99.4\%). Discussion: Telemedicine offers fast and convenient response to patients needs with standardization of triage guidelines. Telemedicine has reduced exposure of the medical workers which reduced the possibility of infection as well as reduction of crowds at the medical centers. Recommendations: Telemedicine is very useful during the COVID-19 pandemic for following up the positive and suspected cases as well as cases with history of close contact with a confirmed positive case.

(c) 2021 The Author(s)

Published by S. Karger AG, Basel

\section{Introduction}

By the end of 2019, a novel coronavirus was initially identified at Wuhan - China and rapidly spread all over the world causing a pandemic with main symptoms of flu-like illness, upper respiratory tract infection, and pneumonia. This pandemic is caused by a new virus called SARS-CoV-2 which was reported after many cases
Correspondence to:

Amr Elhennawy, dr.amr.elhennawy@gmail.com or amelhennawy@dha.gov.ae 
have been diagnosed as viral pneumonia. The most common symptoms are fever and cough; however, other symptoms were reported as loss of taste and/or smell, sore throat, nasal congestion, red eyes, headache, muscle pain, and diarrhea. In severe cases, symptoms include shortness of breath, chest pain, and high temperature [1]. With the easy mode of transmission and rapid rate of infection, a huge number of patients are getting infected every day globally with variety of health conditions. Health-care providers and health-care facilities are facing a big challenge for assessment and accommodation of the large number of patients and providing the needed professional medical care according to the patients' needs. Telemedicine through a telephonic encounter with the patients following a standardized guideline for case triage was helpful in managing the large number of positive cases during the pandemic. With the highly developed healthcare system, technology, and communication infrastructures in Dubai, it was manageable to deal with the large flow of patients on daily basis. One way to overcome this challenge was (Triage Score) as done by the University Hospital in Zurich, who created a triage score to help the doctors at ER to decide if the patient needs hospital admission or could be discharged to home isolation which consists of clinical, radiological, and laboratory findings [2].

A different approach in China was the implementation of telemedicine, which helped to decrease the death rates and provided prevention and treatment instructions and remote consulting for the patients through online tools rather than in-person visit to the clinics or hospitals. Added to this, the National Telemedicine Center of China (NTCC) has established the emergency telemedicine consultation system [3]. In addition to that, the mobility restrictions during the pandemic were problematic for a lot of patients with chronic illness not able to get medical assistance with possibility of deterioration and complications, which could be avoided by telemedicine consultation [4].

The US Centers for Disease Control and Prevention (CDC) have reported a $154 \%$ increase in telehealth remote health care during the last week of March 2020 as compared to the same period last year with multiple benefits to the patients and health-care providers and suggested the continuity of providing telemedicine care after the pandemic [5].

\section{Aim of the Work}

In Dubai during the COVID-19 pandemic, the implementation of telemedicine for patient's assessment and triage was very helpful for managing the large number of positive cases in short time. It was possible to identify the patients' medical needs and provide the needed medical advice and arrange isolation facility or hospital admission according to the patient clinical condition following a standardized guideline. In this study, we wanted to identify the reliability of using the telemedicine as an assessment tool and to detect the accuracy of triage.

\section{Methods}

During the COVID-19 pandemic 2019-2020, Dubai Health Authority has established a central operation room called the COVID-19 Command \& Control Center (CCC) including qualified medical practitioners from different sections aiming to control the pandemic situation by monitoring the situation, analyzing the infection rates and areas, facilitating the PCR tests for the contacts and suspected symptomatic patients, assessment of positive cases by telephonic encounter, and triaging the cases to guide the patients' transfer to isolation facilities or hospitals according to a standardized criteria (DHA Guidelines). The journey starts by tracing the contacts of any confirmed positive case and evaluating the symptomatic cases with flu-like symptoms or upper respiratory tract infection or fever; PCR tests for COVID-19 RNA by nasopharyngeal swabs were arranged, scheduled, and performed at certified labs under Dubai Health Authority.

Confirmed positive cases were informed about their test results by text message to their registered mobile number and later on by receiving a phone call. We used technology to create a database by implementing a system (HASANA System) to register all the positive cases and classify them with complete file of details about the patient's identifications, demographics, and contacts.

A qualified medical team has contacted each patient shortly after the positive swab test results for assurance and giving full instructions for the patients and surroundings at home or workplaces with detailed education about the COVID-19. Assessment of the patients was done by telephonic encounters (Telemedicine) following standardized guidelines; DHA COVID-19 CCC approved guidelines for patient triage focusing on the patient age, fever, symptoms, shortness of breath, comorbidity, etc.

Triage was done according to the gained information; hospital bed arrangement and admission were arranged for moderate to severe cases, while mild and asymptomatic patients were sent to isolation facilities managed by medical teams under Dubai Health Authority. When the patient arrives to hospital or isolation facility, physical examination was performed for reassessment to confirm the patient medical needs. Patient transfer to other isolation facility or medical institute was done accordingly if needed.

In our study, we compared the accuracy of assessment of positive COVID-19 cases by using the telemedicine versus physical examination. Our indicator was the need to transfer the patient to other facility within $12 \mathrm{~h}$ from patient admission to the initially designated facility.

This study is a retrospective, observational, analytical study which was performed by Dubai Health Authority (DHA) staff at the COVID-19 CCC in Dubai - UAE during the COVID-19 pandemic in 2020 among a random sample of confirmed positive cases managed by COVID-19 CCC. Data were obtained from HASA- 
NA database, the sample size was 1,086 cases in a time frame from May to July 2020 with utilization of patient's records at DHA digital files (SALAMA system).

The exclusion criteria included minors below 18 years old and geriatrics above 60 years old, any pregnant women, immunocompromise patients or patients on immunosuppressive medications, patients with physical disability or mental and/or psychological problems, patients managed by institutes other than CCC or admitted to hospital through ER, patients outside the emirate of Dubai or patients on whom physical assessment could not be conducted and finally any symptomatic patients with no COVID-19 confirmed positive PCR test.

We used an investigation tool (questionnaire) for data collection with certain criteria including the age, gender, smoking, BMI, comorbidity, symptoms, action taken, change of action within 12 $\mathrm{h}$, what are the change of action within $12 \mathrm{~h}$ and why the action was changed?

This retrospective study was done by DHA Staff at the DHA COVID-19 CCC in Dubai - UAE during the period from May to July 2020, data collection was done through telephonic encounters, random selection of study sample with no personal details were included in the study and no patients consent was requested.

\section{Statistical Analysis}

Comparison of the outcomes of telemedicine versus physical encounters was done, standard statistical methods were applied. All the patients initially were assessed through telemedicine encounter and accordingly were triaged, the second phase of assessment was physically at the facility. We compared the accuracy of assessment by telemedicine encounter versus physical assessment by detecting any changes of the initial action within $12 \mathrm{~h}$.

\section{Results}

The total number of positive cases included in the study is 1,086 patients, 883 males ( $81.3 \%$ ) and 203 females (18.7\%). The sample included 39 different nationalities, 82 local patients $(7.6 \%)$ while 1,004 expatriate patients (92.4\%).

By analyzing, the patients' medical records we found that 392 patients with BMI below 25 (36.1\%), 460 Patients with BMI between 25 and 30 (42.4\%) while 234 Patients with BMI above 35 (21.5\%) (Table 1).

Among the sample 154 patients were smokers (14.2\%) while 932 patients were nonsmokers (85.5\%), 61 patients were Diabetic (5.6\%) while 1,025 patients were nondiabetic (94.4\%), 91 patients were hypertensive $(8.4 \%)$ while 995 patients were nonhypertensive (91.6\%), 12 patients had previous heart disease (1.1\%), 12 patients had previous pulmonary disease (1.1\%), 22 patients had thyroid problems (2\%) and 5 patients had previous malignancy $(0.5 \%)$ (Table 1$)$.

Regarding the symptoms; 479 patients had no symptoms (44.1\%), 366 patients had fever (33.7\%), 292 patients
Table 1. Patients' medical history

\begin{tabular}{lrr}
\hline & Patients, $n$ & $\%$ \\
\hline BMI & & \\
$\quad$ Normal $(<25)$ & 392 & 36.1 \\
Overweight $(25-30)$ & 460 & 42.4 \\
$\quad$ Obese $(>35)$ & 234 & 21.5 \\
Smoking & & \\
$\quad$ Yes & 154 & 14.2 \\
$\quad$ No & 932 & 85.8 \\
Diabetes & & \\
$\quad$ Yes & 61 & 5.6 \\
$\quad$ No & 1,025 & 94.4 \\
Hypertensive & 91 & 8.4 \\
$\quad$ Yes & 995 & 91.6 \\
$\quad$ No & & \\
Comorbidity & 12 & 1.1 \\
$\quad$ Heart disease & 12 & 1.1 \\
$\quad$ Lung disease & 22 & 0.5 \\
$\quad$ Thyroid & 5 & \\
$\quad$ Malignancy & & \\
\hline
\end{tabular}

Table 2. Patients, symptoms

\begin{tabular}{lcr}
\hline & Patients, $n$ & $\%$ \\
\hline Asymptomatic & 479 & 44.1 \\
Fever & 366 & 33.7 \\
Cough & 292 & 26.9 \\
Sore throat & 149 & 13.7 \\
Difficulty breathing & 101 & 9.3 \\
Loss of smell & 239 & 22 \\
Loss of taste & 251 & 23.1 \\
Diarrhea & 91 & 8.4 \\
\hline
\end{tabular}

had cough (26.9\%), 149 patients had sore throat (13.7\%), 101 patients had difficulty of breathing (9.3\%), 239 patients had loss of smelling (22\%), 251 patients had loss of taste $(23.1 \%)$ while 91 patients had diarrhea (8.4\%) (Table 2).

Initially, 960 Patients were sent to isolation (88.4\%) while 126 patients were admitted to hospitals (11.6\%). The change of action was done only in 27 patients $(2.5 \%), 6$ of them were transferred from isolation to hospital for more investigations or more medical care was required. Twenty patients had transferred from hospital to isolation (7 patients upon their request while 13 other patients were discharged after physical assessment was done to be sent to isolation). One patient was 
Table 3. Initial action and changes of action

\begin{tabular}{lllc}
\hline & $\begin{array}{l}\text { Patients, } \\
n\end{array}$ & $\begin{array}{l}\text { Percentage of } \\
\text { initial action }\end{array}$ & $\begin{array}{c}\text { Percentage of } \\
\text { total number }\end{array}$ \\
\hline Isolation & 960 & - & 88.4 \\
Hospital admission & 126 & - & 11.6 \\
No change of action & $1,059 / 1,086$ & - & 97.5 \\
From isolation to hospital & $6 / 960$ & 0.63 & 0.55 \\
From hospital to isolation & $20 / 126$ & 15.9 & 1.8 \\
From hospital to advanced hospital & $1 / 126$ & 0.79 & 0.09 \\
\hline
\end{tabular}

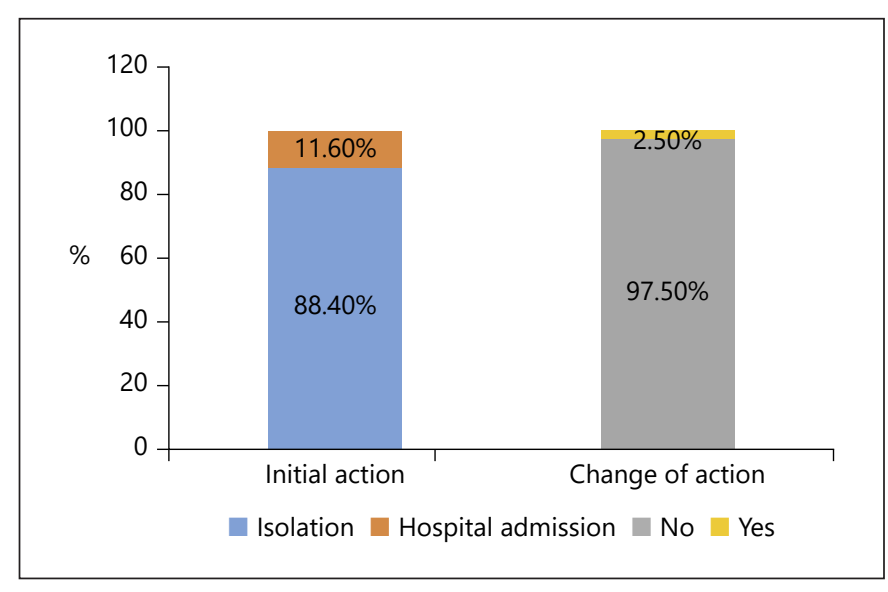

Fig. 1. Initial action and change of action.

transferred from hospital to more advanced hospital as more advanced medical care was required (Table 3; Fig. 1).

\section{Discussion}

One of the most important strategic goals for healthcare sectors during pandemics and disasters is (forward triage) to control the surge and sorting the patients before sending them to Emergency Room (ER) according to their urgency and clinical conditions and what is the expected medical services they might need with a categorized priority according to severity.

With the advanced technology, telemedicine is considered an efficient screening tool which provides protection from exposure to all; patients, clinicians, and community with $24 \mathrm{~h}$ availability to contact the patients by communication tools as telephones, smart phones, and video conferences [6].
In the USA, around 50 health-care sectors already using the telemedicine system during the COVID-19 pandemic to allow clinicians to see patients who are at home such as Jefferson Health's telemedicine practice which has evaluated and treated patients without referring them to ER. Moderate to severe cases as well as high-risk patients were directed for further evaluation by nurse or prepared centers [6].

In our study telemedicine was effective tool for patients triage with high accuracy. 1,059 out of 1,086 patients were directed to the right place from the initial telemedicine assessment (97.5\%). Only 27 patients out of 1,086 screened patients $(2.5 \%)$ were redirected after physical assessment; however, 20 patients of them were downgraded to (stable) after physical assessment with no major medical needs. One patient was already triaged as a hospital case and sent to ER and later on transferred to more advanced hospital setup. The remaining only 6 patients out of 1,086 were upgraded from stable to moderate after physical assessment and were sent to hospital giving the impression that telemedicine was accurate in 1,080 out of 1,086 screened patients $(99.4 \%)$. The remaining 6 patients were not missed as further active monitoring was applied for any possible deterioration of cases.

For all COVID-19 positive cases in Dubai, multiple channels were provided for anyone seeking medical advice with easy and 24/7 availability to communicate with health-care providers at Dubai Health Authority either by calling the call center, using the DHA website, COVID-19 DXB smartphone application, communication with DHA COVID-19 CCC, and multiple assigned emails for notification, case managements, and contact tracing. Multiple Public Health Clinics we assigned and prepared for assessment of COVID-19 positive cases with prior appointment. In case of any emergency, they can go directly to any assigned hospital ER or call the ambulance, a trained medical team we make home assessment. 
Some advantages of telemedicine were the readiness and availability, reduce the delay time for patient assessment, while managing large numbers of cases, reduce exposure of medical staff and community, reduce the crowd at medical centers, and provide service to distant areas at lower costs.

On the other hands, some possible challenges and limitations to establish a telemedicine system include man power and technical training, communication infrastructure, language barriers, patient awareness and compliance, impossible conduction of physical assessment as palpation or auscultation, system shut down, software license, date security, and insurance coverage. Dubai experience of handling the pandemic of COVID-19 pandemic was outstanding with one of the lowest infection and mortality rates all over the world with fast and adaptive recovery.

Multiple modalities and technologies could be used to provide the medical care to patients which could be synchronous by live interaction or asynchronous which allows the patient to record a message to be interpreted later by health-care provider in addition to remote patient monitoring which monitors patient clinical measurements from distance continuously and send it to the health-care providers [7].

Remote monitoring could be used to observe nonurgent conditions for cardiovascular diseases and diabetes, multiple studies included large number of patients have reported the usefulness of using the mobile application to take several pictures for diagnosis of diabetic retinopathy with reliable outcomes [8]. The pandemic has changed the telemedicine practice and rules in many major ways as the physicians would be paid for telemedicine as for in-person visit, and they can attend any patient even from different state if they are licensed at 1 state; however, the physician is allowed to waive or reduced the cost of telemedicine encounter. On the other hand, the patient can attend the encounter while at home or elsewhere [9].

\section{Conclusions}

Telemedicine is the usage of electronic communications tools for providing a remote clinical service to the patients through audio and/or visual communication with health-care provider. Telemedicine is very popular and efficient to manage large number of cases during pandemics/epidemics for fast triage and proper categorization of the large number of patients prior to sending them to the ER to provide the suitable needed medical services on time to the cases in need with limited exposure to the health-care provider and community and avoid unnecessary crowd at the medical centers.

Telemedicine is very useful during the COVID-19 pandemic for following up the positive/suspected cases as well as cases in contact with positive cases. Dubai experience was very successful and useful as a model example for handling the surge efficiently with very low mortality rate.

Telemedicine can be used to monitor and follow up the chronic disease during the pandemic time to reduce the workload at hospitals. Regulations and health authorities should regulate and license and prepare the electronic tools for the telemedicine as it is a very effective tool to provide medical services for our patients even after the subside of COVID-19 pandemic.

\section{Acknowledgements}

We would like to acknowledge all our team members at DHA COVID-19 CCC for their huge efforts.

\section{Statement of Ethics}

This study was approved by Dubai Scientific Research Ethics Committee (DSREC), Dubai Health Authority (DSREC-07/ 2020_43). No personal patients' information was revealed. No specific patient consent was required.

\section{Conflict of Interest Statement}

The authors have no multiplicity of interest to disclose.

\section{Funding Sources}

The authors did not receive any funding.

\section{Author Contributions}

A.E.: primary investigator, study design, collection tool design, data collection and analysis, writing the article and performed the statistics, results, discussion and conclusion - Lead Supervisor and Team Leader. F.A.: contributed to study design. S.B.: Head of Operation Stream. N.A: review and editing. 


\section{References}

1 World Health Organization. Updates 12 October 2020. www.who.int/emergencies/diseases/novel-coronavirus-2019/question-andanswers-hub/q-a-detail/coronavirus-diseasecovid-19\#: :text=symptoms.

2 Levenfus I, Ullmann E, Battegay E, Schuurmans MM. Triage tool for suspected COVID-19 patients in the emergency room: AIFELL score. Braz J Infect Dis. Sep-Oct 2020. 24(5):458-61.

3 Garg S, Galwankar S, Bhatnagar N, Singh MM, Raina SK, Galwankar S. Telemedicine: embracing virtual care during COVID-19 pandemic. J Family Med Prim Care. 2020;9(9):4516-20.
4 Kadir MA. Role of telemedicine in healthcare during the COVID-19 pandemic in the developing countries. TMT. 2020; 5(2):1-5.

5 Koonin LM, Hoots B, Tsang AC, Leroy Z, Farris $\mathrm{K}$, Jolly BT, et al. Trends in the use of telehealth during the emergence of the COVID-19 pandemic: United States, JanuaryMarch 2020. Centers for disease control and prevention (CDC). MMWR Morb Mortal Wkly Rep. 2020 Oct 30;69(43):1595-9.

6 Hollander JE, Carr BG. Virtually perfect? Telemedicine for COVID-19. N Engl J Med. 2020;382:1679-81.
7 Centers for Disease Control and Prevention (CDC). Using telehealth to expand access to essential health services during the COVID-19 pandemic. 2020 Jun 10. https://www. cdc.gov/coronavirus/2019-ncov/hcp/telehealth.html.

8 Galiero R, Pafundi PC, Nevola R, Rinaldi L, Acierno C, Caturano A, et al. The importance of telemedicine during COVID-19 pandemic: a focus on diabetic retinopathy. J Diabetes Res. 2020.

9 Zarefsky M. Five huge ways the pandemic has changed telemedicine. Am Med Assoc. 2020 Aug 26. https://www.ama-assn.org/practicemanagement/digital/5-huge-ways-pandemic-has-changed-telemedicine. 